\section{NEW MINIATURE UNIT}

Dürr Dental has recently launched the VistaScan Mini image plate scanner. This miniature unit is simple to use, does not compromise on image quality and is extremely compact so that it can be easily sited in even the smallest surgery. Scanning and X-ray can literally be at arm's reach.

The re-usable VistaScan image plates are ready within seconds and offer a range of image formats from size 0 to 4. The clarity of the image means clinicians are able to detect caries D1 lesions and endo instruments down to ISO 06.

The unit is easy to integrate and can be operated with DBSWIN or other software packages via plugIns or Twain drivers. VistaScan Mini is ideal for

\section{HANDPIECE CARE}

NSK's Care3 Plus is a simple and reliable way to care for all your high and low speed handpieces. At the touch of a button, Care3 Plus automatically cleans

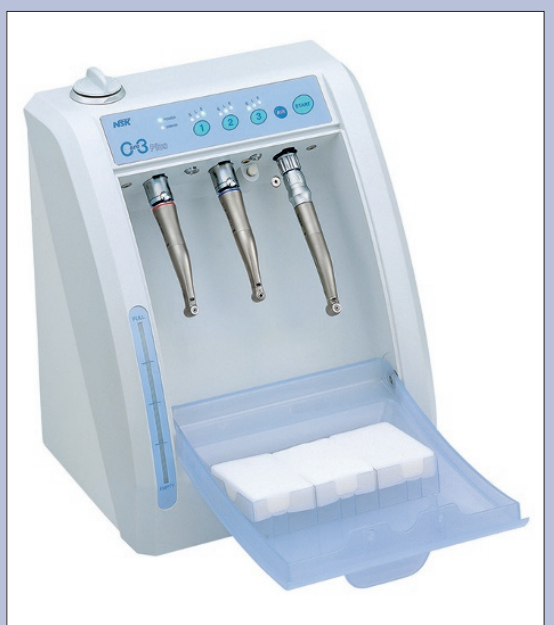

smaller or individual practices without an OPG and can be used in larger surgeries as it obviates the need for the nurse to go out of the room to process plates.

Reader response number 62

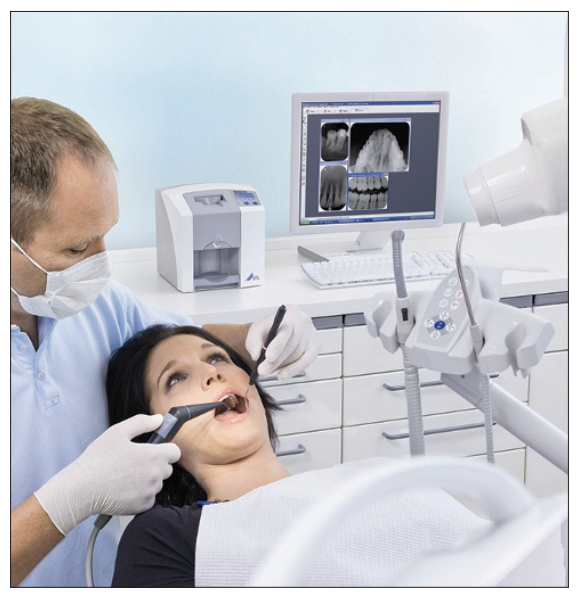

and lubricates up to three instruments at one time, in less than two minutes, ensuring handpiece performance is maintained at all times.

Featuring a unique rotational maintenance system, Care3 Plus ensures all low speed handpieces rotate continuously throughout the cleaning process, guaranteeing that maintenance solution is delivered to even the hardest to reach parts of the handpiece.

Care3 Plus can be used with NSK and all other major brands including $\mathrm{KaVo}$, WEH, Bien Air and Sirona. The unit is available with either a single rotation port and two standard ports or two rotation ports and a single standard port, and with three easy to select cleaning cycles.

Reader response number 63

\section{HIGH QUALITY}

VOICE RECORDING

The new Sony ICD-BX700 and ICD-PX720 digital recorders can be used for taking notes on the go. They feature a high quality $192 \mathrm{kbps}$ MP3 recording mode, up to 280 hours LP recording and a USBto-PC connection feature for the ICD-PX720 model.

With intuitive controls and crisp audio playback, digital voice recorders are designed to make recording easy and hassle-free for busy healthcare professionals. Weighing just $71 \mathrm{~g}$, both recorders come with easy to use controls and crystal clear audio as well as capacity to store up to 280 hours of recording. Operation is quick and easy on both models, thanks to the large buttons and frontal speakers with a full-function display.

Voice activated recording adds convenience and the new $192 \mathrm{kbps}$ MP3 mode ensures clear results with a pin-sharp 20,000 $\mathrm{Hz}$ high frequency range. The playback functions feature digital pitch control so users can tweak playback to their preferred speed and tone, whilst the ability to correct original dictation ensures flawless recordings. Divide and delete editing functions also ensure that the resulting recording is just right.

Both recorders come with $1 \mathrm{~GB}$ of flash memory for file storage, whilst the ICD-PX720 can also be connected to a PC via the USB drive, making for simple file archiving and use with speech-to-text software.

Reader response number 65

\section{PROFESSIONAL REFERRALS MADE EASY}

Specialist dental marketing company Designer Dental has recently launched comprehensive and attractive referral packs for dentists. The contents of a referral pack can vary but the most popular packs produced include an outer folder, referral forms, self addressed envelopes, inserts (eg treatments, fee guide), business cards, case studies/newsletter, a covering letter and outer envelope.

The design of your referral pack can differentiate you from other referral practices. Literature produced by Designer Dental is designed specific to your practice and is completely individual. A high quality and organised image is imperative along with a good use of photography and colours.

Reader response number 64

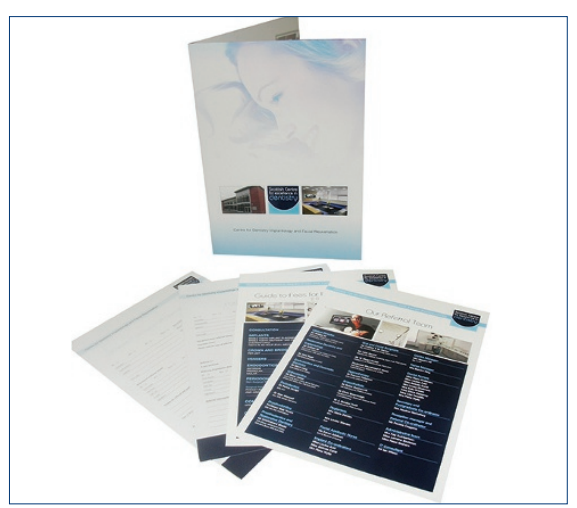

